\title{
Flaw sensitivity of highly stretchable materials
}

\section{Citation}

Chen, Chao, Zhengjin Wang, and Zhigang Suo. 2017. "Flaw Sensitivity of Highly Stretchable Materials." Extreme Mechanics Letters 10 (January): 50-57. doi:10.1016/j.eml.2016.10.002.

\section{Published Version}

doi:10.1016/j.eml.2016.10.002

\section{Permanent link}

http://nrs.harvard.edu/urn-3:HUL.InstRepos:33373175

\section{Terms of Use}

This article was downloaded from Harvard University's DASH repository, and is made available under the terms and conditions applicable to Open Access Policy Articles, as set forth at http:// nrs.harvard.edu/urn-3:HUL.InstRepos:dash.current.terms-of-use\#OAP

\section{Share Your Story}

The Harvard community has made this article openly available.

Please share how this access benefits you. Submit a story.

\section{Accessibility}




\title{
Flaw sensitivity of highly stretchable materials
}

Chao Chen, Zhengjin Wang, and Zhigang Suo (a)

John A. Paulson School of Engineering and Applied Sciences, Kavli Institute for Bionano

Science and Technology, Harvard University, Cambridge, MA 02138, USA

(a) Corresponding author.

Email: suo@seas.harvard.edu

Keywords: Stretchable material; Flaw sensitivity; Rupture; Material-specific length

\begin{abstract}
Elastomers and gels can often deform multiple times their original length. The stretchability is insensitive to small cuts in the samples, but reduces markedly when the cuts are large. We show that this transition occurs when the depth of cut exceeds a material-specific length, defined by the ratio of the fracture energy measured in the large-cut limit and the work to rupture measured in the small-cut limit. This conclusion generalizes a result in the fracture mechanics of hard materials. For an acrylic elastomer and a polyurethane, we measure the stretch to rupture as a function of the depth of cut, and show that the experimental data agree well with the prediction of the nonlinear elastic fracture mechanics. In a space of material properties we compare many materials (elastomers, gels, ceramics, glassy polymers, biomaterials, and metals), and find that the material-specific length varies from nanometers to centimeters.
\end{abstract}




\section{Introduction}

Stretchable materials, such as elastomers and gels, have long been used in tires, seals, gloves, and contact lenses. Under development are new fields of applications, including tissue regeneration [1], drug delivery [2], artificial muscles [3-5], stretchable electronics [5-9], and soft robots $[10,11]$. Stretchable, transparent, ionic conductors (e.g., hydrogels and ionogels) enable devices of unusual functions, such as transparent loudspeakers [12], artificial skins [13], artificial axons $[14,15]$, and electroluminescence of giant stretchability [16-18]. The interest in the mechanics of stretchable materials has surged [19-32].

This paper focuses on a specific issue in the mechanics of stretchable materials: the reduction of stretchability by cuts. A cut can be introduced either intentionally using a razor blade, or unintentionally during fabrication. In the latter case, the cut is commonly called a flaw. The reduction of stretchability by cuts and flaws is called flaw sensitivity. For example, an acrylic elastomer, $\mathrm{VHB}^{\mathrm{TM}}$, commonly used in the development of artificial muscles [3], can deform beyond ten times its original length [33]; however, a VHB sample containing a cut of a few millimeters ruptures when deforming 3-5 times its original length [34]. As another example, a recent tough hydrogel can deform more than twenty times its original length, and a centimeter-long cut reduces the stretchability to seventeen times. [26]

Two approaches exist to predict the rupture of a stretchable device. In one approach, the designer assumes a flawless device, calculates the field of deformation using the nonlinear theory of elasticity, and predicts rupture if any material point in the device reaches a critical state of deformation [35-43]. In the other approach, the designer identifies a flaw in the device, calculates the energy release rate using the nonlinear theory of elasticity, and predicts rupture if the energy release rate reaches the fracture energy [44-47].

The two approaches work well in two limits. The first approach requires no knowledge of flaws, and is applicable in the limit of small flaws. The second approach requires the knowledge of flaws, and is applicable in the limit of large flaws. The transition from flaw-insensitive to flaw- 
sensitive rupture has been discussed in the literature [48-50], but the size of the flaws over which the transition occurs is vague for stretchable materials.

Here we study the transition from flaw-insensitive rupture to flaw-sensitive rupture of highly stretchable materials. For an uncut sample, we measure the work to rupture, $W_{*}$, which has the dimension of energy per unit volume. For a sample with a large cut, we measure the fracture energy, $\Gamma$, which has the dimension of energy per unit area. The ratio of these two parameters, $\Gamma / W_{*}$, defines a material-specific length, which we call the length of flaw sensitivity. Using a combination of experiment and calculation, we show that this material length marks the transition from flaw-insensitive to flaw-sensitive rupture. When the depth of cut $c$ is small compared to $\Gamma / W_{*}$, the stretchability is insensitive to the cut. When $c$ is large compared to $\Gamma / W_{*}$, the stretchability reduces markedly as the depth of the cut increases. Furthermore, we show that flaw sensitivity depends on the stretch-stiffening behavior of elastomers, and that the experimental data agree well with the prediction of nonlinear elastic fracture mechanics. The concept of flaw sensitivity is applicable to all materials, including metals, ceramics, biomaterials, and polymers. We represent the lengths of flaw sensitivity of various materials in a space of material properties, with $W_{*}$ and $\Gamma$ as axes.

The length $\Gamma / W_{*}$ has been used to characterize the intrinsic diameter of the crack tip in elastomers $[51,52]$, but has not been used to characterize flaw sensitivity. We next compare $\Gamma / W_{*}$ to other material lengths commonly used in fracture mechanics. A length, $\Gamma /\left(\sigma_{*}^{2} / E\right)$, appears in the crack-bridging model, where $\sigma_{*}$ is the maximum stress in the traction-separation curve. [53-58] In the crack-bridging model, the region outside bridging zone is linearly elastic with Young's modulus $E$. The work to rupture near the crack tip is given by the $W_{*}=\sigma_{*}^{2} / 2 E$. For highly stretchable materials, however, the material outside the bridging zone is nonlinearly 
elastic. The material length $\Gamma / W_{*}$ generalizes $\Gamma /\left(\sigma_{*}^{2} / E\right)$ when linear elasticity does not apply. (We have dropped any numerical factor.) Another frequently discussed material length is the ratio of fracture energy and elastic modulus, $\Gamma / E$. [32] [59] This length overestimates the length of flaw sensitivity by orders of magnitude. For a highly stretchable material, the stretchability $\lambda_{*}$ is on the order of ten, so that $W_{*} \gg E$. Consequently, the length of flaw sensitivity $\Gamma / W_{*}$ is much smaller than $\Gamma / E$.

\section{Transition from flaw-insensitive to flaw-sensitive rupture}

Flaw-insensitive rupture

To focus on essentials, we consider the stretchability of a thin sheet of a material under a uniaxial force. The length and the width of the sheet are much larger than the thickness of the sheet and the depth of the cut. Using an uncut sample, we measure the applied force as a function of the associated displacement. The area under the force-displacement curve divided by the volume of the material defines the energy density, $W$. Let $\lambda$ be the stretch, namely, the length of the deformed sheet (in the direction of the applied force) divided by the length before stretch. The energy density is a function of stretch, $W(\lambda)$.

For an uncut sample, let $\lambda_{*}$ be the stretch to rupture and $W_{*}$ be the work to rupture. The two parameters are related by the function $W(\lambda)$ :

$$
W_{*}=W\left(\lambda_{*}\right)
$$

The stress to rupture $\sigma_{*}$ is defined by the applied force at rupture divided by the deformed cross-sectional area (perpendicular to the applied force). Criterion (1) also applies to samples containing cuts small compared to a material length (to be specified). 
The stretchability of elastomers is insensitive to small flaws. Table 1 summarizes the experimental data from the literature and from this work. The reported stretch, stress, and work to rupture are within variations $5 \%-20 \%$ of their means. These data were measured using uncut samples. Yet flaws exist inevitably, either as small cracks or as heterogeneities of materials [50]. The small scatter in the data indicates that the stretchability of these materials is insensitive to the small flaws. This observation on elastomers differs from that on brittle hard materials, e.g., silica glass, in which a micron-sized flaw reduces the strength by orders of magnitude [44].

Table 1: Flaw-insensitive rupture of various elastomers.

\begin{tabular}{|c|c|c|c|c|c|}
\hline Source & Material & Stretch & Stress (MPa) & Work $\left(\mathrm{MJ} / \mathrm{m}^{3}\right)$ & Loading Rate \\
\hline Sharma, 1965 [36] & Hycar Aluminum & & 1.035 & & $0.01 \% / \mathrm{s}$ \\
\hline \multirow[b]{2}{*}{ Sharma, 1966 [37] } & Hycar Aluminum & 1.179 & $0.76 \pm 0.14^{*}$ & & $345 \mathrm{~Pa} / \mathrm{s}$ \\
\hline & $\begin{array}{c}\text { Cellulose Acetate } \\
\text { Butyrate }\end{array}$ & 1.096 & 30.2 & & $6.9 \mathrm{kPa} / \mathrm{s}$ \\
\hline \multirow{4}{*}{ Hamdi, 2006 [40] } & $\begin{array}{c}\text { Filled Natural } \\
\text { Rubber }\end{array}$ & $7.12 \pm 0.35^{*}$ & $174.56 \pm 20^{*}$ & $64.55 \pm 12^{*}$ & \multirow{4}{*}{$100 \% / \mathrm{min}$} \\
\hline & $\begin{array}{c}\text { Filled Styrene- } \\
\text { butadiene Rubber }\end{array}$ & $6.88 \pm 0.25^{*}$ & $169.88 \pm 15^{*}$ & $72.07 \pm 18^{*}$ & \\
\hline & $\begin{array}{c}\text { Unfilled } \\
\text { Polyurethane }\end{array}$ & $8.38 \pm 0.6^{*}$ & & & \\
\hline & $\begin{array}{l}\text { Thermoplastic } \\
\text { Elastomer }\end{array}$ & $6.26 \pm 0.5^{*}$ & & & \\
\hline Schmidt, 2012 [33] & VHB & $9.27 \pm 0.23$ & $3.01 \pm 0.47$ & $0.73 \pm 0.07$ & $0.43 \% / \mathrm{s}$ \\
\hline \multirow{2}{*}{ This work } & VHB & $11.93 \pm 0.35$ & $56.8 \pm 16.8^{\#}$ & $5.58 \pm 1.22^{\#}$ & \multirow{2}{*}{$100 \% / \mathrm{min}$} \\
\hline & Polyurethane & $6.08 \pm 0.13$ & $271 \pm 47^{\#}$ & $144 \pm 20^{\#}$ & \\
\hline
\end{tabular}

* The standard deviation estimated through data points in graphs of the source.

\# Data calculated through extrapolating stress-stretch curves to the stretch to rupture based on the Gent model (Fig. 6).

Flaw-sensitive rupture

Flaw-sensitive rupture is predicted by fracture mechanics [47]. Consider a sheet containing a cut. The elastic energy of the sample is a function $U(\Delta, c)$, where $c$ is the depth of the cut in the undeformed state, and $\Delta$ is the displacement associated with the applied force. 
The reduction in the elastic energy when the cut extends a unit area defines the energy release rate, $G=-\partial U(\Delta, c) /(t \partial c)$, where $t$ is the thickness of the sheet in the undeformed state. The energy release rate can be determined by solving the boundary-value problem of nonlinear elasticity. When other sizes of the specimen are much larger than the cut, the depth of cut $c$ is the only length scale in the boundary-value problem. Dimensional considerations dictate that the energy release rate should take the form, $G(\lambda, c)=k(\lambda) W(\lambda) c$, where $k(\lambda)$ is a dimensionless function determined by solving the boundary-value problem. The function $k(\lambda)$ depends on the model of nonlinear elasticity $[47,60]$. The sample ruptures at stretch $\lambda_{R}$ when the energy release rate reaches the fracture energy, $G\left(\lambda_{R}, c\right)=\Gamma$. When the cut is large and the sample ruptures at a small applied strain, $\lambda \rightarrow 1$, linear elasticity applies, and the smalldeformation limit for an edge cut is known, $k(1) \approx 2(1.1215)^{2} \pi \approx 7.9$ [61]. The criterion of rupture becomes

$$
W\left(\lambda_{R}\right)=\frac{\Gamma}{k(1) c} .
$$

This result is the Griffith limit [44]. Thus, we characterize the flaw-sensitive rupture by the fracture energy $\Gamma$ in the limit of large flaws.

\section{The transition from flaw-insensitive rupture to flaw-sensitive rupture}

We have characterized the rupture of an uncut sample by the work to rupture, $W_{*}$, which has the dimension of energy per unit volume. For a sample containing a cut, the state $W_{*}$ prevails ahead the front of the cut when the sample is near rupture. We have also characterized the rupture in the limit of large cuts by the fracture energy, $\Gamma$, which has the dimension of 
energy per unit area. The ratio of these two material parameters defines a material-specific length, $\Gamma / W_{*}$.

We argue that the material length $\Gamma / W_{*}$ marks the transition from flaw-insensitive to flaw-sensitive rupture. When a sample of a small cut ruptures, except for the unstrained region behind the front of the cut, the entre sample reaches the state of $W_{*}$ (Fig. 1a). When a sample of a large cut ruptures, only a small zone around the front of the cut reaches the state of $W_{*}$ (Fig. 1b). Inside this zone, fracture process occurs. Outside this zone, the field of deformation is well characterized by the nonlinear theory of elasticity. The length scale of the fracture process zone is estimated as follows. A dimensional consideration dictates that energy density $W$ should scale with the energy release rate $G$, and inversely scale with the distance to crack tip $r$, namely, $W \sim G / r$. This scaling appears in the analytical solutions of the nonlinear elastic field around the front of cut. [25] When the sample ruptures, the energy release rate $G$ reaches $\Gamma$, and the energy density $W$ in the fracture process zone attains $W_{*}$. Consequently, the size of the fracture process zone scales with the material length $\Gamma / W_{*}$.

A flaw-sensitivity diagram displays the stretch to rupture $\lambda_{R}$ as a function of the depth of cut $c$ (Fig. 1c). The transition occurs when the depth of cut $c$ is comparable to the material length $\Gamma / W_{*}$. When the depth of the cut $c$ is small compared to $\Gamma / W_{*}$, the stretch to rupture is insensitive of the depth of the cut, and the small-cut limit (1) applies. When the depth of cut is large compared to the material length, the stretch to rupture decreases as the depth of the cut increases, and approaches the large-cut limit (2). 

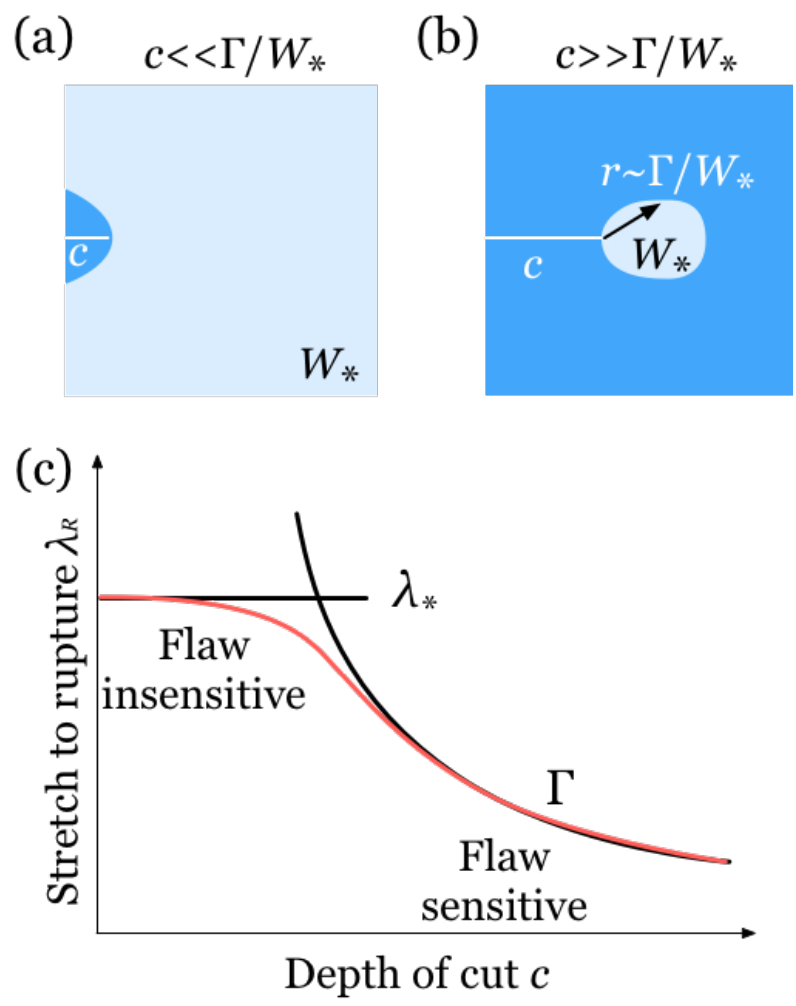

Fig. 1. The transition from flaw-insensitive to flaw-sensitive rupture. (a) When a sample of a small cut ruptures, except for the region behind the front of the cut, the entire sample reaches the state of $W_{*}$. (b) When a sample of a large cut ruptures, only a small zone around the front of the cut reaches the state of $W_{*}$. The size of this zone scales with the material length $\Gamma / W_{*}$. (c) A flaw-sensitivity diagram sketches the stretch to rupture as a function of the depth of cut. The diagram shows the small-cut limit, the large-cut limit, and the transition between the two limits.

The material length $\Gamma / W_{*}$, together with the depth of the cut $c$, defines a dimensionless number:

$$
\chi=\frac{c}{\Gamma / W_{*}}
$$

which we call the number of flaw sensitivity. We write the relation between the stretch to rupture $\lambda_{R}$ and the depth of the cut $c$ in the form:

$$
W\left(\lambda_{R}\right)=W_{*} f(\chi) .
$$


The function $f(\chi)$ approaches the two limits: the small-cut limit $f \rightarrow 1$ as $\chi \rightarrow 0$, and the large-cut limit $f \rightarrow(\chi k(1))^{-1}$ as $\chi \rightarrow \infty$. The transition occurs at $\chi \sim 1$. Many functions satisfy these requirements. As an illustration, we propose a function,

$$
W\left(\lambda_{R}\right)=\frac{W_{*}}{1+k(1) c W_{*} / \Gamma},
$$

which conforms to the form of (4), matches the two limits (1) and (2), and marks the transition at $c \sim \Gamma / W_{*}$. However, to predict the exact flaw-sensitivity diagram, we need to model the fracture process and the nonlinear elastic deformation.

\section{Experimental determination of flaw-sensitivity diagrams}

We measure the flaw-sensitivity diagrams of two elastomers: an acrylic elastomer VHB $4905\left(3 \mathrm{M}^{\mathrm{TM}}\right)$ and a polyurethane (PU, 90A Durometer), both purchased from McMASTERCARR. In experiment, we pull a dogbone-shaped sample with a cut to rupture by a uniaxial force. We measure the stretch to rupture $\lambda_{R}$ as a function of the depth of cut $c$.

To prevent rupture at the loading fixture, we prepare samples in the shape of a dogbone, so that the stress is high in the narrow region of the sample, away from the loading fixture (Fig. 2a). We use a razor blade to cut the sample to a depth $c$ ranging from $\sim 0.05 \mathrm{~mm}$ to $50 \mathrm{~mm}$. We mark two lines close to the ends of narrow region with distance $L$. The thickness of the sheet $t=$ $0.5 \mathrm{~mm}$. The width of the dogbone $w$ is at least 5 times the depth of cut $c$, and the distance $L$ is at least twice the width $w$, varying case by case. When the cut is small, we take a photo to measure its depth (Fig. 2f). The sticky VHB sample is rolled to two steel rods. We also roll the rods during pulling to further alleviate stress concentration at the installation region (Fig. 2c). The PU samples are knotted to the steel rods (Fig. 2d). Then we pull the specimen at a strain rate of $100 \%$ per minute (Fig. 2b). The stretch of ligament to rupture is $\lambda_{R}$. During stretching, 
the initial sharp cut (Fig. 2f) blunts with a visible growth of millimeters before rupture (Fig. 2g). This stable growth of cut gives rise to the fracture resistance curve.

(a)

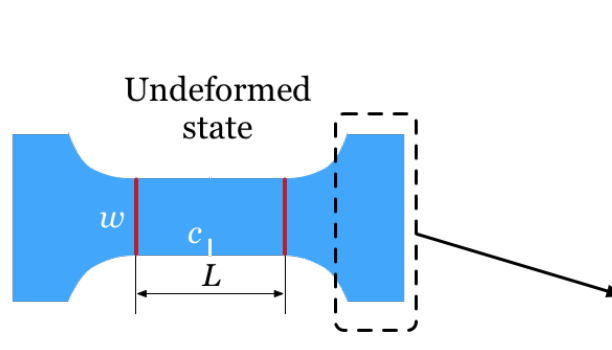

(b)
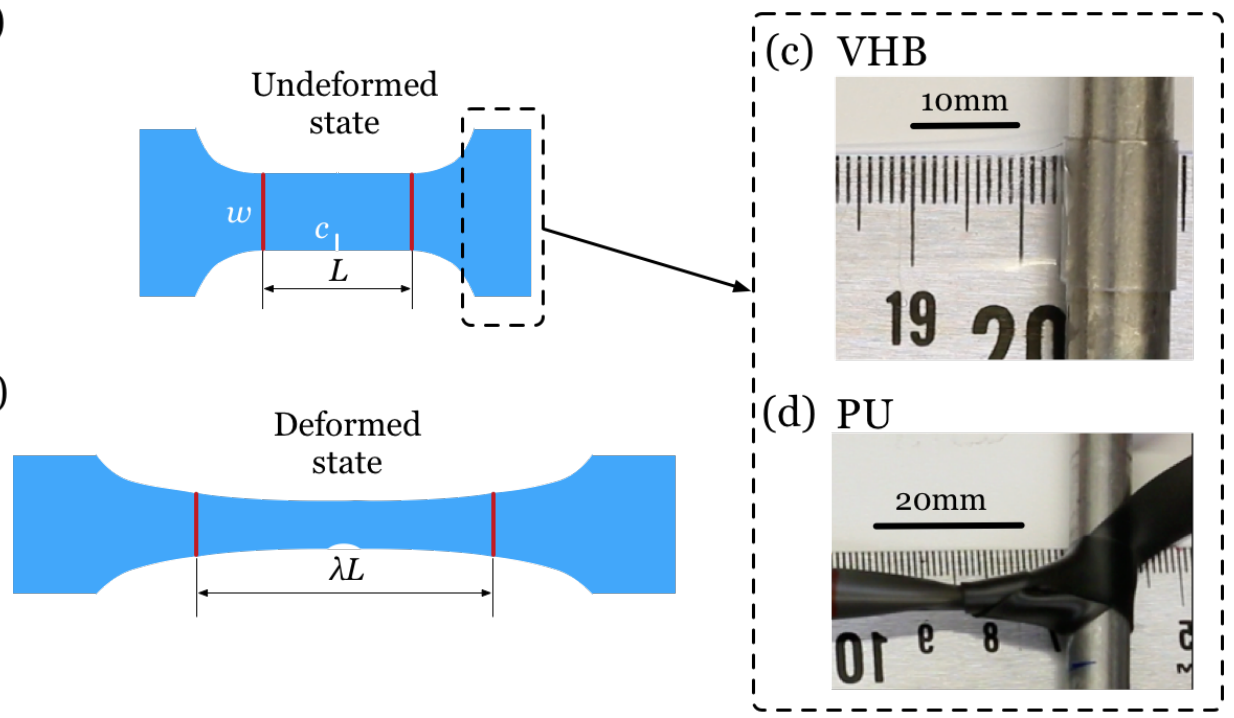

(e)

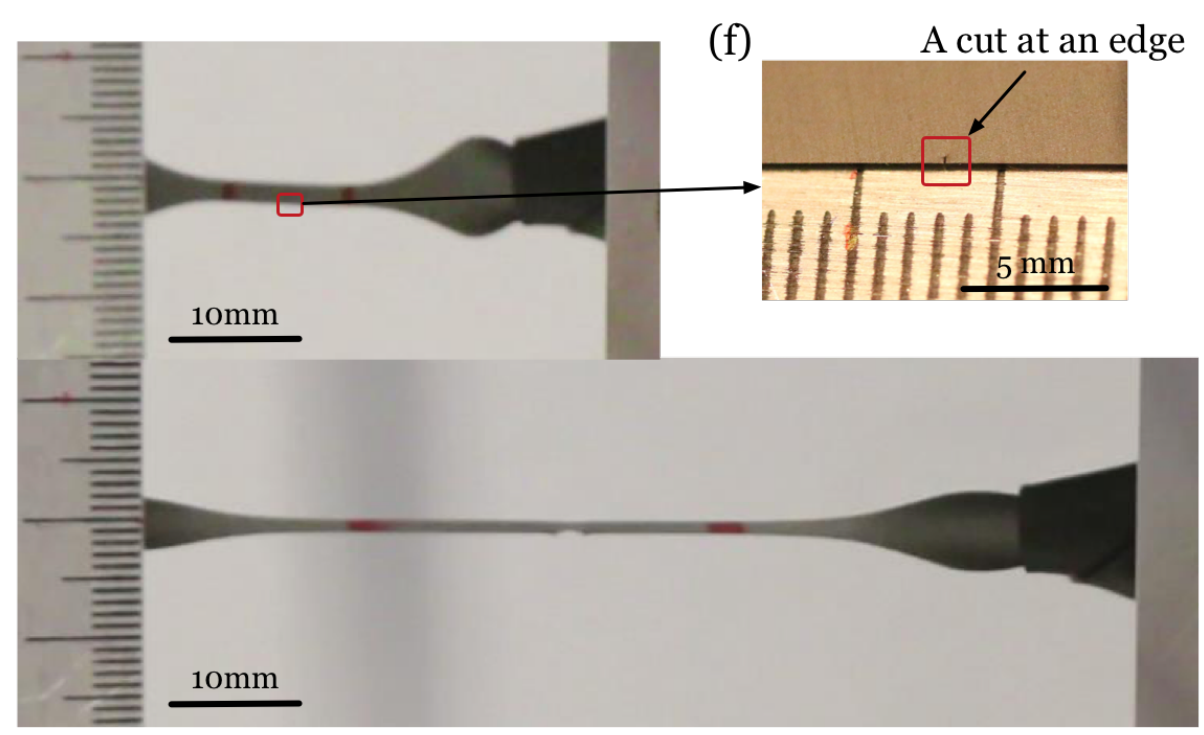

Fig. 2. The experiment to determine the flaw-sensitivity diagrams of a VHB and a PU. (a) In the undeformed state, a dogbone-shaped sample is cut to depth $c$. Two red lines mark the region of uniform width $w$ and length $L$. (b) When the sample is stretched by a uniaxial force, the distance between the marked lines becomes $\lambda L$. (c) The sticky VHB is rolled to steel rods. (d) The PU with slippery surface is knotted to the steel rods. (e) A photo of a cut PU sample before stretching. (f) A photo of a submillimeter cut before stretching. (g) A photo of the stretched PU sample. The cut blunts, and the growth of the cut before rupture is visible. 
(a)

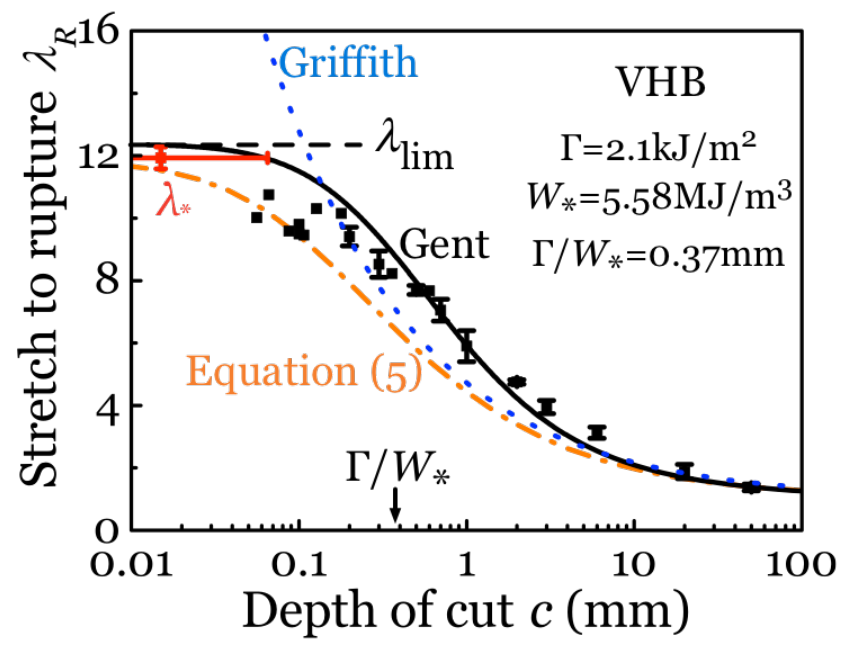

(b)

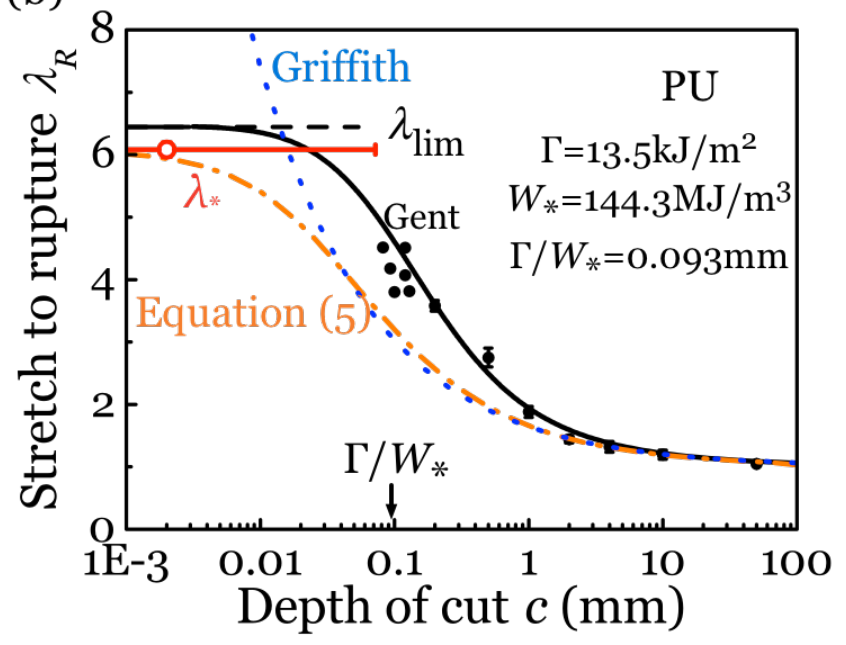

Fig. 3. Flaw-sensitivity diagrams of two elastomers: (a) a VHB and (b) a PU. In experiments, dogbone-shaped specimens are cut to a certain depth and pulled to rupture. Each run of the experiment records a stretch to rupture $\lambda_{R}$. The error bar shows the standard deviation of stretches to rupture with at least three measurements. The scatter shows the stretch to rupture when the depth of cut is smaller than $0.2 \mathrm{~mm}$. The experimental data are compared to the prediction of the nonlinear elastic fracture mechanics (marked as Gent), and to the fitting formula (marked as Equation (5)). The stretches to rupture of uncut samples are marked red. The vertical error bar is the standard deviation of ten measurements, and the horizontal error bar is an estimation of the range of flaw size.

We plot the measured stretch to rupture $\lambda_{R}$ as a function of the depth of cut $c$ (Fig. 3). The error bars for ruptures with $c \geq 0.2 \mathrm{~mm}$ are the standard deviations of at least three 
measurements with the same depth. When the depth $c$ is smaller than $0.2 \mathrm{~mm}$, we are unable to control the depth of cut precisely. We show the scatters directly.

Also included in Fig. 3 are the stretches to rupture determined using uncut samples. The experimental setup is similar to that described previously, but no cut is introduced before stretching. The statistics of stretch to rupture involves ten tests. The vertical error bar indicates the standard deviation of these measurements. Since there is no intentional cut in the specimens, the flaws exist in microscale, smaller than the minimum depth of intentional cut. As an illustration, the horizontal error bar shows the range of flaw size.

We include the limits of small flaws and large flaws in Fig. 3. In the small-flaw limit, the work to rupture, $W_{*}$, characterizes rupture of specimens without an intentional cut. To obtain $W_{*}$, we measure stress-stretch curves, fit them to the Gent model (9) (Fig. 6, described later), and estimate $W_{*}=W\left(\lambda_{*}\right)$. The work to rupture is $5.58 \mathrm{MJ} / \mathrm{m}^{3}$ for $\mathrm{VHB}$, and $144.3 \mathrm{MJ} / \mathrm{m}^{3}$ for PU. In the large-flaw limit, we fit the measured stretch to rupture to the Griffith theory (2) to determine the fracture energy. The fracture energy is $2.1 \mathrm{~kJ} / \mathrm{m}^{2}$ for $\mathrm{VHB}$ and $13.5 \mathrm{~kJ} / \mathrm{m}^{2}$ for PU. The ratio $\Gamma / W_{*}$ gives the lengths of flaw sensitivity: $0.37 \mathrm{~mm}$ for $\mathrm{VHB}$, and $0.093 \mathrm{~mm}$ of PU. We label the values of $\Gamma / W_{*}$ on the horizontal axes in Fig. 3, showing $\Gamma / W_{*}$ agrees with the transition of flaw sensitivity in the experiment.

Flaw sensitivity is evident through comparison between experimental data and the two limits (Fig. 3). When the depth of cut $c$ is large compared to $\Gamma / W_{*}$, the cut significantly reduces the stretch to rupture $\lambda_{R}$. In the large-flaw limit, when rupture occurs at a small applied strain, the stretch to rupture reduces to the Griffith limit (2). When the stretch of rupture is beyond small strain, the flaw-sensitive rupturing stretches deviate from (2). We will elaborate this effect in the next section using nonlinear elastic fracture mechanics. In the small-flaws limit, the stretch to ruptures approaches a constant $\lambda_{*}$ with small variations; rupture is flaw insensitive, 
and (1) is applicable. The interpolation of the two limits, Equation (5), of course works well in the two limits, but underestimates the stretches to rupture for samples containing cuts of intermediate depths.

\section{Flaw-sensitivity determined by nonlinear elastic fracture mechanics}

When the cut in a sample is not very deep, the prediction of the Griffith theory deviates from the experimental data (Fig. 3). This deviation is not surprising. To predict the flawsensitivity diagram accurately, we need a model of fracture process, as well as a model of nonlinear elasticity with large deformation. A model of fracture process is beyond the scope of this paper. Here we focus on the effect of nonlinear elasticity.

A stretchable material is a three-dimensional network of crosslinked polymer chains. Each polymer chain is an entropic spring. [62] When the elastomer is under no external forces, the chain coils to maximize its entropy. When the elastomer is subject to external forces, the chain uncoils, and eventually stiffens upon approaching its contour length. Macroscopically, this behavior yields a stiffening stress-stretch curve, and the stress becomes unbounded at a limiting stretch. Arruda and Boyce [63] developed an eight-chain model, which shows that the energy density $W$ is solely determined by one invariant of the deformation,

$$
I=\lambda_{1}^{2}+\lambda_{2}^{2}+\lambda_{3}^{2}-3
$$

where $\lambda_{1}, \lambda_{2}$, and $\lambda_{3}$ are the principal stretches. The shear modulus of a stretchable material is often orders of magnitude lower than bulk modulus. Subject to external forces, the material distorts much more than it dilates. Consequently, the stretchable material is assumed to be incompressible,

$$
\lambda_{1} \lambda_{2} \lambda_{3}=1
$$

Particularly, stretched by the uniaxial force to $\lambda$, the invariant of deformation $I=\lambda^{2}+2 \lambda^{-1}-3$. We adopt a functional form of the energy density proposed by Gent [64]: 


$$
W(I)=-\frac{E I_{\lim }}{6} \log \left(1-\frac{I}{I_{\lim }}\right) .
$$

This model only involves two material parameters: Young's modulus $E$ at small strain, and $I_{\lim }$, the limit of the invariant $I$ in (6).

The material model of chosen elastomers is identified in Section 6. For each material, we measure the stress-stretch curve and fit it to the Gent model (Fig. 6). Both VHB and polyurethane show significant stiffening effect when stretch approaches to limit. The stressstretch measurements confirm that the Gent model is capable to describe the deformation of chosen elastomers (Fig. 6).

We use a finite element software, ABAQUS, to calculate the energy release rate. ABAQUS does not support the Gent model directly, and we use the UHYPER subroutine to implement the Gent model in ABAQUS. We calculate the energy release rate using the $J$-integral. [65] The geometry is a large sheet with a cut. The cut exists at the middle of one edge with depth $c$, which is $1 / 10$ of the width of sheet $w$, and $1 / 20$ of length $L$ (Fig. 4a). We assume the thin sheet in plane stress condition. Because of the symmetry of the geometry, we model a half of the sheet with a symmetric boundary in crack plane. To avoid the singularity at the crack tip in calculations, we model a blunt tip with a small radius $\rho=c / 1000$ before stretching. We use the CPS8R element in ABAQUS (Fig. 4b). The calculations choose the material parameter $I_{\text {lim }}$ according to experiments of the chosen materials, i.e., $I_{\text {lim }}=150$ for VHB, and 39 for PU. (Fig. 6) The normalized energy release rate $G /(E c)$ is plotted as the applied stretch $\lambda$ (Fig. 4c).

We determine the stretch to rupture by fracture mechanics. With the prescribed fracture energy of material, $\Gamma$, we pull the sheet to stretch $\lambda$ by a uniaxial force. When the energy release rate reaches fracture energy, $G=\Gamma$, the corresponding stretch to rupture $\lambda_{R}$ is determined. 

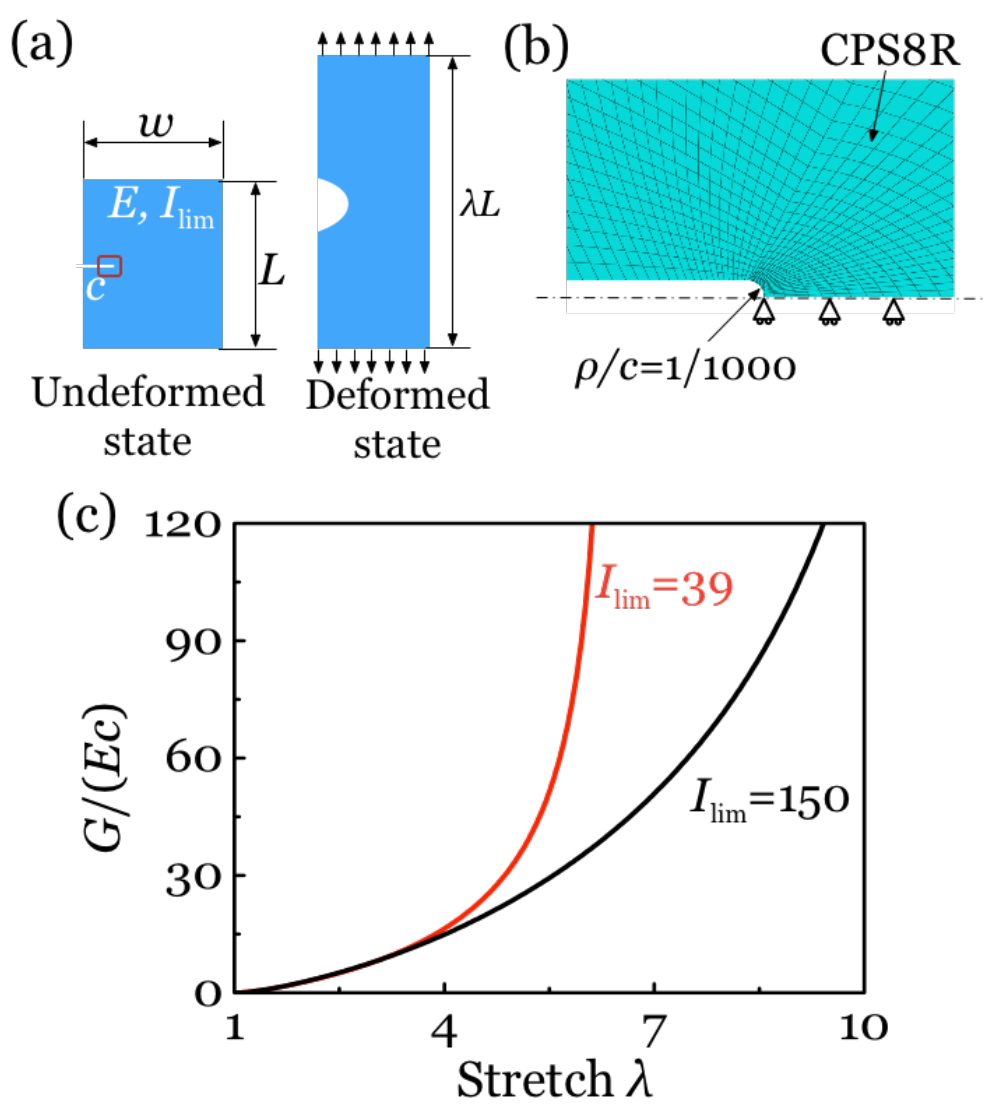

Fig. 4. Finite element calculations of energy release rate of the Gent materials. (a) Schematics of a sheet with a cut. In the undeformed state, the depth of the cut is $c$, the width of the sheet $w$ $=10 c$, and the length $L=20 c$. In the deformed state, the sheet is pulled to a length $\lambda L$ by a uniaxial force. (b) Finite radius $\rho$ of the tip. The mesh of the sheet is modeled by the CPS8R element. (c) The normalized energy release rate $G /(E c)$ is plotted as a function of the applied stretch $\lambda$.

We include the finite element results in Fig. 3. When rupture occurs at large deformation, the nonlinear elastic fracture mechanics predicts flaw-sensitive rupture, while the linear Griffith prediction (2) deviates from experiment. When rupture occurs at small strain, the nonlinear prediction reduces to Griffith limit (2). The limiting stretch $\lambda_{\lim }$ of the Gent model is also marked as the dashed horizontal lines, but is slightly higher than the stretch to rupture $\lambda_{*}$ in the small-cut limit. 
The discrepancy in the small-cut limit is not surprising. The limiting stretch $\lambda_{\lim }$ is determined by fitting the stress-stretch curve to the Gent model, and is a parameter to describe the elasticity of the material. On the other hand, $\lambda_{*}$ is the stretch to rupture measured using uncut samples. The rupture process is distinct from elastic behavior. Elastic behavior represents a homogeneous stretching of polymer chains, which share load fairly. But when the material is stretched to its limits, short chains carry load more. Thus rupture behavior is dominated mostly by weaker chains, and deviates from elastic prediction.

\section{Flaw sensitivity of various materials}

The notion of flaw sensitivity applies to all materials. We collect data of fracture energy $\Gamma$ and work to rupture $W_{*}$ of various materials, e.g. ceramics, polymers, biomaterials, metals, etc. The fracture energy is the required work to propagate a crack front by unit area. Some literature reports fracture toughness, $K_{C}$. In this case, we convert to fracture energy through $\Gamma=K_{C}^{2} / E$. The area under the stress-stretch curve to failure defines the work to rupture $W_{*}$.

This parameter is not commonly reported in literatures. An estimation is $W_{*}=\alpha \sigma_{*}\left(\lambda_{*}-1\right)$, where $\alpha$ is a numerical factor depending on the shape of the stress-stretch curve. When the majority of the stress-stretch curve is linear, $\alpha \sim 1 / 2$, and $W_{*} \sim \sigma_{*}^{2} / 2 E$. When the stress-stretch curve shows a long plateau, $\alpha \rightarrow 1$. Particularly, the work to rupture for brittle hard materials is estimated through the theoretical bond energy of covalent bonds.

We plot the data for various materials in a material space with $W_{*}$ and $\Gamma$ as axes (Fig.5). The dashed slashes mark the constant values of the length of flaw sensitivity $\Gamma / W_{*}$. The length of flaw sensitivity has a large range, from nanometers for brittle materials to centimeters for tough materials. 


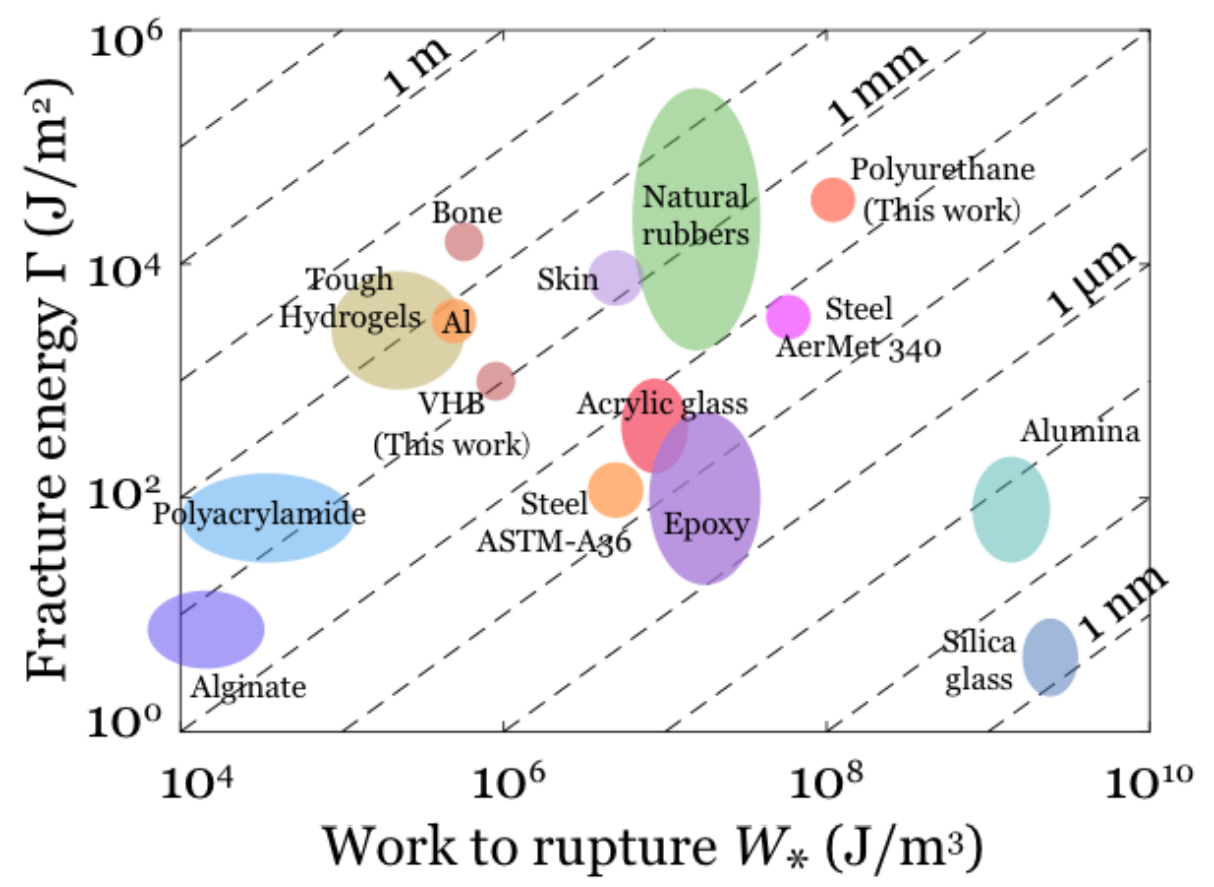

Fig. 5. A space of material properties, with the two axes being the fracture energy $\Gamma$ and the work to rupture $W_{*}$. Also included are the slashes of constant values of the length of flaw sensitivity $\Gamma / W_{*}$. The stretchable materials in the current work are compared with other materials, e.g., natural rubbers $[47,66]$, polyacrylamide hydrogels [26], alginate hydrogels [26, 67], and tough hydrogels, [26, 68], as well as steels, aluminum, bone, human skin, acrylic glass, epoxy, aluminum oxide, and silica glass. [69]

For brittle hard solids (e.g., a silica glass), measuring $\sigma_{*}$ and $W_{*}$ in the small-flaw limit is a difficult experimental task, and is rarely done in practice, because the small-flaw limit is reached when the flaws approach the atomic scale. In practice, brittle hard solids nearly always operate in the large-flaw limit, where the Griffith fracture mechanics applies. By contrast, for elastomers and gels, the small-flaw limit is readily reached when the flaws are below millimeters. In practice, elastomers and gels can operate in the small-flaw limit, the large-flaw limit, and anywhere in between. As we have commented before, the scatter of the rupture data measured using uncut samples is large for brittle hard solids, but is small for elastomers and gels.

Additional insight into the length of flaw sensitivity is gained by comparing two ideal models of fracture. In these ideal models, the work to rupture $W_{*}$ is the energy per unit volume 
stored in the chemical bonds in a material. The rupture of an ideal hard solid (e.g., silica glass) dissipates the chemical energy of a layer of atomic bonds, so that $\Gamma \approx W_{*} a$, where $a$ is length of the chemical bond, corresponding to the thickness of the atomic layer. By contrast, the rupture of an ideal elastomer dissipates the chemical energy of a layer of polymer chains, so that $\Gamma \approx W_{*} a \sqrt{n}$, where $a$ is the length of a monomer, and $n$ is the number of monomers in the chain. [70] Consequently, the length of flaw sensitivity $\Gamma / W_{*}$ is estimated by the atomic size $a$ for an

ideal hard solid, and by the polymeric mesh size $a \sqrt{n}$ for an ideal elastomer. These ideal models neglect other mechanisms of dissipation and underestimate the fracture energy. But these models do bring out a fundamental cause for the large difference in the lengths of flaw sensitivity of the two types of materials.

\section{Stress-stretch curves}

We measure the stress-stretch curves of the two elastomers. The experimental setup is similar with the measurement of flaw-sensitivity diagrams. We prepare samples in the shape of a dogbone. The thickness of sheet is $t=0.5 \mathrm{~mm}$. The width of the dogbone ligament is $w=1 \mathrm{~mm}$. The area of cross-section is $A=w t$. We mark two lines close to the ends of the uniform ligament with a distance $L=10 \mathrm{~mm}$. To prevent slip from the grips, the sticky VHB sample is rolled to two steel rods, while the smooth PU samples are knotted to steel rods. Then we pull the samples uniaxially in an Instron machine. The pulling force $F$ is recorded in Instron. Simultaneously we take videos to record the distance of between the lines $l$. The nominal stress is $F / A$, and the stretch of the ligament $\lambda$ is $l / L$. We plot the nominal stress as a function of stretch in Fig. 6. The area under the curve gives the energy density as a function of the stretch, $W(\lambda)$.

Under the uniaxial tensile force, the Gent model predicts the relation between the nominal stress and the stretch: 


$$
\frac{F}{A}=\frac{E\left(\lambda^{2}-\lambda^{-1}\right)}{3\left(1-\frac{\lambda^{2}+2 \lambda^{-1}-3}{I_{\lim }}\right)} .
$$

The experimental data fit to the Gent model with $E=84 \mathrm{kPa}$ and $I_{\lim }=150$ for VHB, and $E=10.5$ MPa and $I_{\text {lim }}=39$ for PU (Red dashed lines in Fig. 6). The fitting of PU is excellent in the entire range of stretch. The fitting of VHB underestimates the stress when the strain is small. Yet the sacrifice of fitting accuracy at small strain contributes to a better overall match, and captures the stress-stiffening effect.

(a)

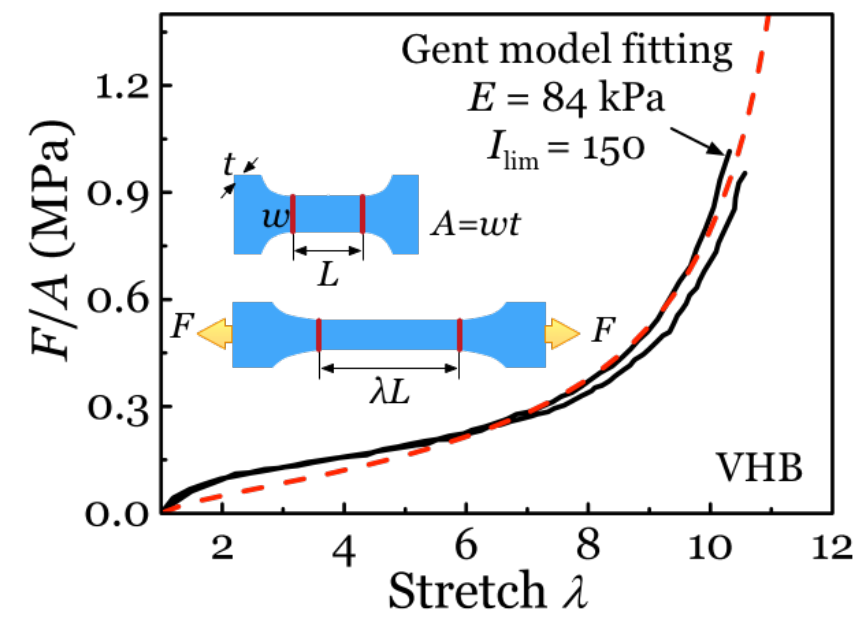

(b)

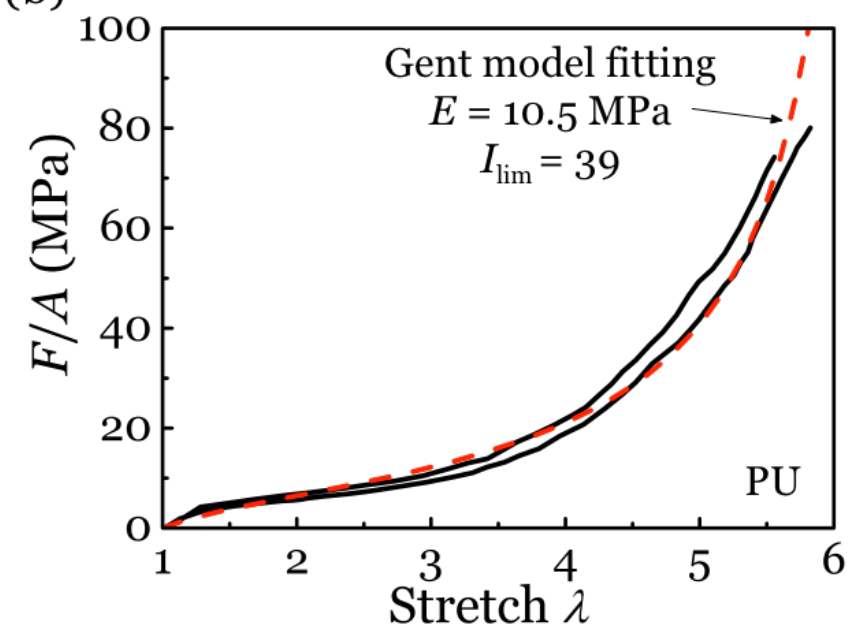


Fig. 6. Stress-stretch curves of a VHB and a PU under uniaxial forces. The samples are in the shape of a dogbone. In undeformed state, the ligament in the sample is with uniform width $w$. The thickness of sample is $t=0.5 \mathrm{~mm}$. The cross-sectional area is $A=w t$. The distance between marked lines is $L=10 \mathrm{~mm}$. Subject to an uniaxial force $F$, the distance between lines becomes $\lambda L$. The experimental stress-stretch curves (solid curves) fit to the Gent model (dashed curves), giving the material parameters: $E$ and $I_{\text {lim. }}$. (a) Stress-stretch relation of VHB $(w=2 \mathrm{~mm})$, (b) and of PU $(w=1 \mathrm{~mm})$.

\section{Concluding remarks}

This work analyzes the flaw sensitivity of highly stretchable materials. We measure the work to rupture $W_{*}$ using uncut samples, and measure the fracture energy $\Gamma$ using samples containing large cuts. We identify a length of flaw sensitivity, $\Gamma / W_{*}$. A combination of experiment and calculation shows that the stretch to rupture is nearly a constant when the depth of cut is small compared to $\Gamma / W_{*}$, and reduces markedly when the depth of cut is large compared to $\Gamma / W_{*}$. Furthermore, we represent the stiffening stress-stretch curves to the Gent model, and use the finite element method to calculate the energy release rate. The nonlinear elastic fracture mechanics predicts the transition from flaw-insensitive to flaw sensitive rupture. The length of flaw sensitivity is a property of materials of various kinds, varying from nanometers to centimeters. We display these data in a material property space with $W_{*}$ and $\Gamma$ as axes.

\section{Acknowledgements}

Work is supported by MRSEC (DMR 14-20570). We thank Professors David Mooney for the use of Instron machine. We thank Philipp Rothemund and Guoyong Mao for the helpful discussions on rupture tests.

\section{References}

1. Marler J.J., Upton J., Langer R., and Vacanti J.P., Transplantation of cells in matrices for tissue regeneration. Advanced Drug Delivery Reviews, 1998. 33(1-2): p. 165-182. 
2. Lee K.Y. and Mooney D.J., Hydrogels for tissue engineering. Chemical Reviews, 2001. 101(7): p. 1869-1879.

3. Pelrine R., Kornbluh R., Pei Q., and Joseph J., High-speed electrically actuated elastomers with strain greater than 100\%. Science, 2000. 287(5454): p. 836-839.

4. Anderson I.A., Gisby T.A., and McKay T.G., Multi-functional dielectric elastomer artificial muscles for soft and smart machines. Journal of Applied Physics, 2012. 112(4): p. 041101.

5. Kim D.H., Lu N., Ma R., Kim Y.S., Kim R.H., Wang S., Wu J., Won, Tao H., Islam A., Yu K.J., Kim T.i., Chowdhury R., Ying M., Xu L., Li M., Chung H.J., Keum H., McCormick M., Liu P., Zhang Y.W., Omenetto F.G., Huang Y., Coleman T., and Rogers J.A., Epidermal electronics. Science, 2011. 333(6044): p. 838-843.

6. Minev I.R., Musienko P., Hirsch A., Barraud Q., Wenger N., Moraud E., Gandar J., Capogrosso M., Milekovic T., Asboth L., Torres R., Vachicouras N., Liu Q., Pavlova N., Duis S., Larmagnac A., Vörös J., Micera S., Suo Z., Courtine G., and Lacour S.P., Electronic dura mater for long-term multimodal neural interfaces. Science, 2015. 347(6218): p. 159-163.

7. Lee S., Reuveny A., Reeder J., Lee S., Jin H., and Liu Q., A transparent bendinginsensitive pressure sensor. Nature Nanotechnology, 2016. 11(5): p. 472-478.

8. Lipomi D.J., Tee B., Vosgueritchian M., and Bao Z., Stretchable organic solar cells. Advanced Materials, 2011. 23(15): p. 1771-1775.

9. $\quad$ Lipomi D.J., Vosgueritchian M., Tee B., Hellstrom S.L., Lee J.A., Fox C.H., and Bao Z., Skin-like pressure and strain sensors based on transparent elastic films of carbon nanotubes. Nature Nanotechnology, 2011. 6(12): p. 788-792.

10. Shepherd R.F., Ilievski F., Choi W., Morin S.A., Stokes A.A., Mazzeo A.D., Chen X., Wang M., and Whitesides G.M., Multigait soft robot. Proceedings of the National Academy of Sciences, 2011. 108(51): p. 20400-20403.

11. Zhao H., Li Y., Elsamadisi A., and Shepherd R., Scalable manufacturing of high force wearable soft actuators. Extreme Mechanics Letters, 2015. 3: p. 89-104.

12. Keplinger C., Sun J.-Y., Foo C., Rothemund P., Whitesides G.M., and Suo Z., Stretchable, transparent, ionic conductors. Science, 2013. 341(6149): p. 984-987.

13. Sun J.-Y., Keplinger C., and Whitesides G.M., Ionic skin. Advanced Materials, 2014. 26(45): p. 7608-7614.

14. Yang C., Chen B., Lu J., Yang J., Zhou J., Chen Y., and Suo Z., Ionic cable. Extreme Mechanics Letters, 2015. 3: p. 59-65.

15. Robinson S.S., O'Brien K.W., Zhao H., and Peele B.N., Integrated soft sensors and elastomeric actuators for tactile machines with kinesthetic sense. Extreme Mechanics Letters, 2015. 5: p. 47-53.

16. Yang C.H., Chen B., Zhou J., and Chen Y.M., Electroluminescence of giant stretchability. Advanced Materials, 2015. 28(22): p. 4480-4484.

17. Larson C., Peele B., Li S., Robinson S., and Totaro M., Highly stretchable electroluminescent skin for optical signaling and tactile sensing. Science, 2016. 351(6277): p. 1071-1074.

18. Wang J., Yan C., Chee K., and Lee P., Highly stretchable and self deformable alternating current electroluminescent devices. Advanced Materials, 2015. 27(18): p. 2876-2882.

19. Baumberger T., Caroli C., and Martina D., Solvent control of crack dynamics in a reversible hydrogel. Nature Materials, 2006. 5(7): p. 552 - 555. 
20. Gong J.P., Why are double network hydrogels so tough? Soft Matter, 2010. 6(12): p. 2583-2590.

21. Cheng S.H. and Sun C.T., Size-dependent fracture toughness of nanoscale structures: Crack-tip stress approach in molecular dynamics. Journal of Nanomechanics and Micromechanics, 2014. 4(4): p. A4014001.

22. Suo Z., Theory of dielectric elastomers. Acta Mechanica Solida Sinica, 2010. 23(6): p. 549-578.

23. Zhao X., Multi-scale multi-mechanism design of tough hydrogels: building dissipation into stretchy networks. Soft Matter, 2014. 10(5): p. 672-687.

24. Lin S., Zhou Y., and Zhao X., Designing extremely resilient and tough hydrogels via delayed dissipation. Extreme Mechanics Letters, 2014. 1: p. 70-75.

25. Long R. and Hui C.Y., Crack tip fields in soft elastic solids subjected to large quasistatic deformation-A review. Extreme Mechanics Letters, 2015. 4: p. 131-155.

26. Sun J.-Y., Zhao X., Illeperuma W.R.K., Chaudhuri O., and Oh K.H., Highly stretchable and tough hydrogels. Nature, 2012. 489(7414): p. 133-136.

27. Ducrot E., Chen Y., Bulters M., Sijbesma R.P., and Creton C., Toughening elastomers with sacrificial bonds and watching them break. Science, 2014. 344(6180): p. 186-189.

28. Crosby A.J., Shull K.R., Lakrout H., and Creton C., Deformation and failure modes of adhesively bonded elastic layers. Journal of Applied Physics, 2000. 88(5): p. 2956.

29. Segura T., Anderson B.C., Chung P.H., and Webber R.E., Crosslinked hyaluronic acid hydrogels: A strategy to functionalize and pattern. Biomaterials, 2005. 26(4): p. 359-371.

30. Shull K.R., Flanigan C.M., and Crosby A.J., Fingering instabilities of confined elastic layers in tension. Physical Review Letters, 2000. 84(14): p. 3057-3060.

31. Volokh K.Y. and Vorp D.A., A model of growth and rupture of abdominal aortic aneurysm. Journal of Biomechanics, 2008. 41(5): p. 1015-1021.

32. Creton C. and Ciccotti M., Fracture and adhesion of soft materials: A review. Reports on Progress in Physics, 2016. 79(4): p. 46601.

33. Schmidt A., Rothemund P., and Mazza E., Multiaxial deformation and failure of acrylic elastomer membranes. Sensors and Actuators A: Physical, 2012. 174: p. 133-138.

34. Pharr M., Sun J.-Y., and Suo Z., Rupture of a highly stretchable acrylic dielectric elastomer. Journal of Applied Physics, 2012. 111(10): p. 104114.

35. Smith T.L., Ultimate tensile properties of elastomers. II. Comparison of failure envelopes for unfilled vulcanizates. Journal of Applied Physics, 1964. 35(1): p. 27-36.

36. Sharma M.G. and Lim C.K., Experimental investigations on fracture of polymers. Polymer Engineering and Science, 1965. 5(4): p. 254-262.

37. Sharma M.G., Failure of polymeric materials under biaxial stress fields. Polymer Engineering and Science, 1966. 6(1): p. 30-35.

38. Smith T.L. and Rinde J.A., Ultimate tensile properties of elastomers. V. Rupture in constrained biaxial tensions. Journal of Polymer Science Part A - 2: Polymer Physics, 1969. 7(4): p. 675-685.

39. Dickie R.A. and Smith T.L., Ultimate tensile properties of elastomers. VI. Strength and extensibility of a styrene-butadiene rubber vulcanizate in equal biaxial tension. Journal of Polymer Science Part A - 2: Polymer Physics, 1969. 7(4): p. 687-707.

40. Hamdi A., Abdelaziz N.M., Hocine A.N., Heuillet P., and Benseddiq N., A fracture criterion of rubber-like materials under plane stress conditions. Polymer Testing, 2006. 25(8): p. 994-1005. 
41. Volokh K., Hyperelasticity with softening for modeling materials failure. Journal of the Mechanics and Physics of Solids, 2007. 55(10): p. 22372264.

42. Trapper P. and Volokh K.Y., Elasticity with energy limiters for modeling dynamic failure propagation. International Journal of Solids and Structures, 2010. 47(25-26): p. 33893396.

43. Hamdi A. and Mahjoubi H., Design criterion for rubbery parts under biaxial loading. Theoretical and Applied Fracture Mechanics, 2015. 78: p. 51-55.

44. Griffith A.A., The phenomena of rupture and flow in solids. Philosophical Transactions of the Royal Society of London A, 1921. 221: p. 163-198.

45. Irwin G.R. Fracture dynamics. in Fracturing of Metals, ASM Symposium (Trans. ASN 40A). 1948. Cleveland.

46. Orowan E., Fracture and strength of solids. Reports on Progress in Physics, 1948. 12: p. 185-232.

47. Rivlin R.S. and Thomas A.G., Rupture of rubber. I. Characteristic energy for tearing. Journal of Polymer Science, 1953. 10(3): p. 291-318.

48. Setua D.K. and De S.K., Effect of short fibres on critical cut length in tensile failure of rubber vulcanizates. Journal of Materials Science, 1985. 20(7): p. 2653-2660.

49. Akhtar S., Bhowmick A.K., De P.P., and De S.K., Tensile rupture of short fibre filled thermoplastic elastomer. Journal of Materials Science, 1986. 21(12): p. 4179-4184.

50. Hamed G.R., Effect of crosslink density on the critical flaw size of a simple elastomer. Rubber Chemistry and Technology, 1983. 56(1): p. 244-251.

51. Thomas A.G., Rupture of rubber. II. The strain concentration at an incision. Journal of Polymer Science, 1955. 18(88): p. 177-188.

52. Volokh K.Y. and Trapper P., Fracture toughness from the standpoint of softening hyperelasticity. Journal of the Mechanics and Physics of Solids, 2008. 56(7): p. 24592472.

53. Dugdale D.S., Yielding of steel sheets containing slits. Journal of the Mechanics and Physics of Solids, 1960. 8(2): p. 100-104.

54. Bilby, Cottrell A.H., and Swinden K.H., The spread of plastic yield from a notch. Proceedings of the Royal Society A: Mathematical, Physical and Engineering Sciences, 1963. 272(1350): p. 304-314.

55. Cottrell A.H., Mechanics of fracture, in Symposium on Fracture. 1963: Univerisity of Melbourne. p. 1-27.

56. Bao G. and Suo Z., Remarks on crack-bridging concepts. Applied Mechanics Reviews, 1992. 45(8): p. 355-366.

57. Hui C.Y., Ruina A., Long R., and Jagota A., Cohesive zone models and fracture. The Journal of Adhesion, 2011. 87(1): p. 1-52.

58. Hui C.Y., A J., Bennison S.J., and Londono J.D., Crack blunting and the strength of soft elastic solids. Proceedings of the Royal Society A, 2003. 459(2034): p. 1489-1516.

59. Knowles J.K. and Sternberg E., On a class of conservation laws in linearized and finite elastostatics. Archive for Rational Mechanics and Analysis, 1971. 44(3): p. 187-211.

60. Greensmith H.W., Rupture of rubber. XI. Tensile rupture and crack growth in a noncrystallizing rubber. Journal of Applied Polymer Science, 1964. 8(3): p. 1113-1128.

61. Tada H., Paris P., and Irwin G., The stress analysis of cracks handbook ASME. New York, 2000: p. 55-56.

62. Treloar L.R.G., Physics of Rubber Elasticity. 1975, USA: Oxford University Press. 
63. Arruda E.M. and Boyce M.C., A three-dimensional constitutive model for the large stretch behavior of rubber elastic materials. Journal of the Mechanics and Physics of Solids, 1993. 41(2): p. 389-412.

64. Gent A.N., A new constitutive relation for rubber. Rubber Chemistry and Technology, 1996. 69(1): p. 59-61.

65. Rice J.R., A path independent integral and the approximate analysis of strain concentration by notches and cracks. Journal of Applied Mechanics, 1968. 35(2): p. 1726.

66. Wegst U.G.K. and Ashby M.F., The mechanical efficiency of natural materials. Philosophical Magazine, 2004. 84(21): p. 2167-2186.

67. Drury J.L., Dennis R.G., and Mooney D.J., The tensile properties of alginate hydrogels. Biomaterials, 2004. 25(16): p. 3187-3199.

68. Yang C., Wang M., Haider H., Yang J., Sun J.-Y., Chen Y., Zhou J., and Suo Z., Strengthening alginate/polyacrylamide hydrogels using various multivalent cations. ACS Applied Materials \& Interfaces, 2013. 5(21): p. 10418-10422.

69. Li J., Illeperuma W.R.K., Suo Z., and Vlassak J.J., Hybrid hydrogels with extremely high stiffness and toughness. ACS Macro Letters, 2014. 3(6): p. 520-523.

70. Lake G.J. and Thomas A.G., The strength of highly elastic materials. Proceedings of the Royal Society A, 1967. 300(1460): p. 108-119. 